\title{
A framework for optimization of turbulent wind-farm boundary layers and application to optimal control of wind-farm energy extraction
}

\author{
Johan Meyers, Wim Munters, and Jay Goit
}

\begin{abstract}
A PDE-based optimization framework is presented that allows optimization of turbulent wind-farm boundary layers. It consists of a state-of-the-art large-eddy simulation code that allows the time-resolved simulation of the threedimensional turbulent flow in the atmospheric boundary layer, together with the adjoint (backward) sensitivity equations to this nonlinear system of PDEs (i.e. the incompressible Navier-Stokes equations). Both the forward and the backward system are efficiently parallelized for supercomputing, and are combined with state-of-the-art gradient-based optimization methods. We use this tool to investigate the use of optimal coordinated control of wind-farm boundary-layer interaction with the aim of increasing the total energy extraction in wind farms. The individual wind turbines are considered as flow actuators and their energy extraction is dynamically regulated in time so as to optimally influence the flow field. Earlier work on wind-farm optimal control in the fully developed regime (Goit \& Meyers 2015, J. Fluid Mech. 768, 550) is discussed, and extended towards wind farms in which inflow effects are important.
\end{abstract}

\section{INTRODUCTION}

It is well know that wake accumulation, and the interaction of the wind farm with the atmospheric boundary layer leads to a decrease in energy extraction downstream in the farm that can amount up to $40 \%$ and more [1], [2]. The current work investigates coordinated optimal control of wind turbines in a wind farm, focussing on improving energy extraction. Individual turbines are considered as flow actuators, whose energy extraction can be regulated dynamically in time and per turbine. To this end, our main focus is on the control of the wind turbine's axial induction factor by means of the thrust coefficient. In the past, studies on increasing energy extraction in wind farms by means of induction control have mainly focused on optimization of quasi-static power set-points of individual turbines throughout the farm (cf. [3]-[8]), that are only slowly adapted to changing atmospheric conditions (e.g. at a rate of once per 15 minutes). The main idea is that downrating the power output from upwind turbines in a farm, so that the wind speed in their wake would be higher, leads to higher energy extraction in downwind turbines, and possibly an overall increase of power extraction. These studies all relied on simple wake

Johan Meyers, Wim Munters, and Jay Goit are with the Department of Mechanical Engineering, KU Leuven, 3001 Leuven, Belgium. Email: johan.meyers@kuleuven. be. The authors acknowledge support from the European Research Council (FP7-Ideas, grant no. 306471), the Flemish Science Foundation (FWO, grant no. G.0376.12), and BOF KU Leuven (grant no. IDO/11/012). Simulations were performed on the computing infrastructure of the VSC Flemish Supercomputer Center, funded by the Research Foundation Flanders (FWO) and the Flemish Government department EWI. engineering models to test and develop their ideas, lacking at that time the means to validate against experiments or highfidelity turbulence-resolving flow simulations (such as LES). Recently, Annoni et al. [8] performed a detailed analysis using large-eddy simulations. Unfortunately, they find that static downrating of upstream turbines is not effective, as the reduction of wake deficit diffuses too much to be sufficiently captured by the downstream turbines. Similar findings were also observed in recent wind-tunnel testing (L. Sætran, J. Bartl, NTNU Norway - pers. comm.).

To date, research into much faster dynamic changes of power set-points for increase of energy extraction has not received much attention, though some work has focused on wind-farm power tracking in combination with load reduction [9], [10]. Set points may be adapted at much faster rates than typically considered in quasi-steady approaches, e.g. 10 to 20 seconds, so that the turbine directly interacts with turbulent flow structures, possibly influencing wake mixing and entrainment. The challenge in developing and evaluating such dynamic wind-farm control approaches is related to the very high dimensionality and complexity of the turbulent flow state with which the controls should interact. In addition, simulating the evolution of this turbulent flow state with large-eddy simulations is very expensive, while any other faster wake models are based on implicit assumptions on wake and wind-farm boundary-layer dynamics. As a result designing controllers based on intuition or simple first-principle based physical insights is nontrivial. The lack of success of static set-point optimization discussed above, illustrates this point.

In the current work, we focus on improving energy extraction of wind farms in large-eddy simulations (LESs), where the LES model itself is used as a control model in a recedinghorizon optimal-control framework. Such an approach is infeasible as a real wind-farm controller, as computational costs are exhibitive. Instead, the methodology is used as a means to explore the potential of coordinated control in wind farms, without excluding a priori any of the turbulent flow physics that could potentially improve performance. Recently, this approach was used by Goit \& Meyers [11] for fully developed 'infinite' wind-farm boundary layers. To this end, they employed gradient-based optimization of the control actions, using adjoint LES to estimate the highdimensional gradients of the optimal-control objective functional. They found that energy extraction could be potentially increased by up to $16 \%$. However, the limit of 'infinite' wind farms is representative only for the fully developed boundary-layer part of very large wind farms. Such a regime 
typically arises for wind farms with a streamwise extend that is larger than 10 to $20 \mathrm{~km}$, in which the energy extraction in the wind farm is governed by the vertical transport of kinetic energy towards the turbine region [12]. Most modern wind farm are not that large, or at least have a major portion of their turbines situated in the first $10 \mathrm{~km}$. In this region, the atmospheric boundary layer is still developing and the upstream inflow of kinetic energy still plays an important factor in overall performance. In the current work, we extend the approach by Goit \& Meyers [11] to a regular 'finite' wind farm, in which entrance effects in the first rows are expected to play an important role.

\section{COMPUTATIONAL METHOD}

\section{A. Forward simulation model}

Large-eddy simulations of wind-farm boundary layers are performed in SP-Wind, an in-house research code that was developed in a series of earlier studies on wind-farm simulations, and flow optimization (see e.g. Ref. [13]-[15]). The governing equations are the filtered incompressible Navier-Stokes equations for neutral flows and the continuity equation, i.e.

$$
\begin{aligned}
\nabla \cdot \widetilde{\boldsymbol{u}} & =0 \\
\frac{\partial \widetilde{\boldsymbol{u}}}{\partial t}+\widetilde{\boldsymbol{u}} \cdot \nabla \widetilde{\boldsymbol{u}} & =-\frac{1}{\rho} \nabla \widetilde{p}+\nabla \cdot \boldsymbol{\tau}_{M}+\boldsymbol{f}
\end{aligned}
$$

where $\widetilde{\boldsymbol{u}}=\left[\widetilde{u}_{1}, \widetilde{u}_{2}, \widetilde{u}_{3}\right]$ is the resolved velocity field, $\widetilde{p}$ is the pressure field. For the subgrid-scale model $\boldsymbol{\tau}_{M}$ a standard Smagorinsky model [16] is used, combined with Mason \& Thomson's wall damping [17] at the wall. Furthermore, $f$ represents the forces (per unit mass) introduced by the turbines on the flow. They are modelled using an actuatordisk model (ADM) and written for turbine $i$ as

$$
\boldsymbol{f}^{(i)}=-\frac{1}{2} C_{T, i}^{\prime} \widehat{V}_{i}^{2} \mathscr{R}_{i}(\boldsymbol{x}) \boldsymbol{e}_{\perp} \quad i=1 \cdots N_{t},
$$

where $C_{T, i}^{\prime}$ is the disk-based thrust coefficient (note that $C_{T, i}^{\prime}$ differs from the conventional thrust coefficient $C_{T}$, which uses undisturbed inflow as a reference - see Refs. [11], [12] for details). Further, $\widehat{V}_{i}$ is the disk-averaged and time filtered the disk velocity, i.e. [12], [15], [18]

$$
\begin{aligned}
\frac{\mathrm{d} \widehat{V}_{i}}{\mathrm{~d} t} & =\frac{1}{\tau}\left(V_{i}-\widehat{V}_{i}\right), \\
V_{i}(t) & =\frac{1}{A} \int_{\Omega} \widetilde{\boldsymbol{u}}(\boldsymbol{x}, t) \cdot \boldsymbol{e}_{\perp} \mathscr{R}_{i}(\boldsymbol{x}) \mathrm{d} \boldsymbol{x} .
\end{aligned}
$$

Here, $\mathscr{R}_{i}(\boldsymbol{x})$ is a geometrical smoothing function that distributes the uniform surface force of the turbine over surrounding LES grid cells, and $\boldsymbol{e}_{\perp}$ represents the unit vector perpendicular to the turbine disk. Finally, for the time filter (4), we use $\tau=5 \mathrm{~s}$. For more details regarding the implementation of the ADM in the SP-Wind, the reader is referred to Meyers \& Meneveau [15], and Goit \& Meyers [11].

\section{B. Optimization problem}

We consider a classical receding-horizon optimal control approach. In this approach, a control time horizon $T$ is selected, and the control parameters are optimized as a function of time over this control horizon given the full interaction with the turbulent flow field as described by the LES equations. Here, the control parameters $\varphi(t)$ correspond to all disk-based turbine thrust coefficients $\varphi \equiv$ $\left[C_{T, 1}^{\prime}(t), C_{T, 2}^{\prime}(t), \cdots, C_{T, N_{t}}^{\prime}(t)\right]$. The aim is to maximize the total wind-farm energy extraction. Thus, we consider following cost functional

$$
\begin{aligned}
\mathscr{J}(\boldsymbol{\varphi}, \boldsymbol{q}) & =-\int_{0}^{T} P(t) \mathrm{d} t=\int_{0}^{T} \int_{\Omega} \boldsymbol{f} \cdot \widetilde{\boldsymbol{u}} \mathrm{d} \boldsymbol{x} \mathrm{d} t \\
& =-\int_{0}^{T} \sum_{i=1}^{N_{t}} \frac{1}{2} C_{\mathrm{T}, \mathrm{i}}^{\prime} \widehat{V}_{i}^{2} V_{i} \mathrm{~d} t
\end{aligned}
$$

We further introduce the notation $\boldsymbol{q} \equiv[\widetilde{\boldsymbol{u}}(\boldsymbol{x}, t), \widetilde{p}(\boldsymbol{x}, t), \widehat{\boldsymbol{V}}(t)]$ for the state (with $\widehat{\boldsymbol{V}}=\left[\widehat{V}_{1}, \cdots, \widehat{V}_{N_{t}}\right]$ ). Also, the state equations $(1,2,4)$ are written in short-hand notation as $\boldsymbol{B}(\boldsymbol{\varphi}, \boldsymbol{q})=0$. Finally, we use the notation $\boldsymbol{q}(\boldsymbol{\varphi})$ for the solution to the state equations given the control inputs $\varphi$, such that $\boldsymbol{B}(\boldsymbol{\varphi}, \boldsymbol{q}(\boldsymbol{\varphi})) \equiv 0$.

The optimization problem is now defined as

$$
\min _{\boldsymbol{\varphi}, \boldsymbol{q}} \mathscr{J}(\boldsymbol{\varphi}, \boldsymbol{q}) \quad \text { s.t. } \boldsymbol{B}(\boldsymbol{\varphi}, \boldsymbol{q})=0 \text {. }
$$

To solve this problem, we formulate it in it's reduced form (similar to a single shooting approach), by introducing $\tilde{J}(\varphi) \equiv \mathscr{J}(\varphi, \boldsymbol{q}(\varphi))$, so that we end up with an unconstrained optimization problem $\min _{\varphi} \tilde{\mathscr{J}}(\varphi)$. This problem is solved using a Polak-Ribière conjugate-gradient method in combination with the Brent line search algorithm [19]-[21]. See Ref. [11] for details.

\section{Gradient and backward simulation model}

The gradient of the reduced cost functional can be derived as (cf. Ref. [11] for details)

$$
\nabla \tilde{\mathscr{J}}=\frac{\partial \mathscr{J}}{\partial \varphi}+\left[\frac{\partial \boldsymbol{B}}{\partial \varphi}\right]^{*} \boldsymbol{q}^{*}
$$

with $[\partial \boldsymbol{B} / \partial \boldsymbol{\varphi}]^{*}$ the adjoint of $\partial \boldsymbol{B} / \partial \boldsymbol{\varphi}$, and $\boldsymbol{q}^{*}=(\boldsymbol{\xi}, \pi, \boldsymbol{\chi})$ the solution of the adjoint equations (see further below). For the current cost functional (6), this leads to [11]

$$
\begin{aligned}
\nabla \tilde{\mathscr{J}} & =\frac{\partial \mathscr{J}}{\partial \boldsymbol{\varphi}}+\frac{1}{2} \int_{\Omega} \widehat{\boldsymbol{V}}^{\circ 2} \circ \mathscr{R}(\mathbf{x})\left[\boldsymbol{\xi} \cdot \boldsymbol{e}_{\perp}\right] \mathrm{d} \boldsymbol{x} \\
& =\frac{1}{2} \int_{\Omega} \widehat{\boldsymbol{V}}^{\circ 2} \circ \mathscr{R}(\mathbf{x})\left[(-\widetilde{\boldsymbol{u}}+\boldsymbol{\xi}) \cdot \boldsymbol{e}_{\perp}\right] \mathrm{d} \boldsymbol{x}
\end{aligned}
$$

with $\mathscr{R} \equiv\left[\mathscr{R}_{1}, \cdots, \mathscr{R}_{N_{t}}\right]$, and where $\circ$ is used to denote the entry-wise product (or Hadamard product), and $\widehat{\boldsymbol{V}}^{\circ 2}$ is the entry-wise square of $\widehat{\boldsymbol{V}}$.

The adjoint solution, required for the evaluation of Eq. (10) is obtained by solving following adjoint wind-farm 


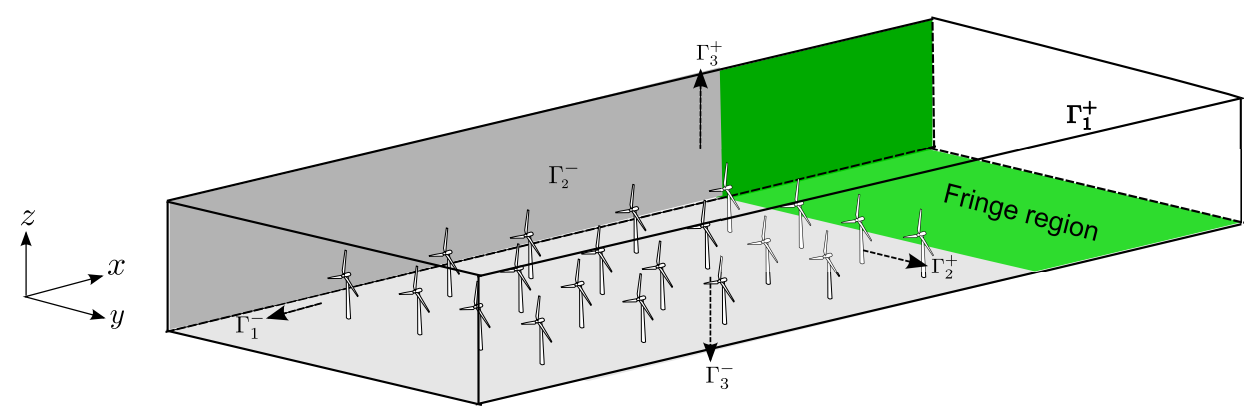

Fig. 1: Computational domain with the fringe region.

LES equations (cf. Ref. [11] for details of the derivation)

$$
\begin{aligned}
& -\frac{\partial \boldsymbol{\xi}}{\partial t}-\widetilde{\boldsymbol{u}} \cdot \nabla \boldsymbol{\xi}-(\nabla \boldsymbol{\xi})^{T} \cdot \widetilde{\boldsymbol{u}}=-\frac{1}{\rho} \nabla \pi+\nabla \cdot \boldsymbol{\tau}_{M}^{*}+\boldsymbol{f}^{*} \\
& \nabla \cdot \boldsymbol{\xi}=0 \\
& -\frac{\mathrm{d} \chi_{i}}{\mathrm{~d} t}=\frac{1}{\tau}\left[-\chi_{i}+C_{T, i}^{\prime} \widehat{V}_{i} \int_{\Omega} \mathscr{R}_{i}(\mathbf{x})(\widetilde{\boldsymbol{u}}-\boldsymbol{\xi}) \cdot \boldsymbol{e}_{\perp} \mathrm{d} \boldsymbol{x}\right], \\
& \quad \text { for } i=1 \cdots N_{t} .
\end{aligned}
$$

Here, $(\boldsymbol{\xi}, \pi, \boldsymbol{\chi})$ are the adjoint variables associated to each state variable $\boldsymbol{q}=(\widetilde{\boldsymbol{u}}, \widetilde{p}, \widehat{\boldsymbol{V}})$. Further, $\boldsymbol{f}^{*}$, and $\boldsymbol{\tau}_{M}^{*}$ are the adjoint forcing term, and the adjoint of the SGS model respectively. They are given by

$$
\begin{aligned}
\boldsymbol{f}^{*} & =\sum_{i=1}^{N_{t}}\left(\frac{1}{2} C_{T, i}^{\prime} \widehat{V}_{i}^{2}+\frac{\chi_{i}}{A}\right) \mathscr{R}_{i}(\boldsymbol{x}) \boldsymbol{e}_{\perp} \\
\boldsymbol{\tau}_{M}^{*} & =2 \ell_{s}^{2}\left(\frac{2 \boldsymbol{S}: \boldsymbol{S}^{*}}{(2 \boldsymbol{S}: \boldsymbol{S})^{1 / 2}} \boldsymbol{S}+(2 \boldsymbol{S}: \boldsymbol{S})^{1 / 2} \boldsymbol{S}^{*}\right),
\end{aligned}
$$

where $\boldsymbol{S}^{*}=\left(\nabla \boldsymbol{\xi}+(\nabla \boldsymbol{\xi})^{T}\right) / 2, \boldsymbol{S}=\left(\nabla \boldsymbol{u}+(\nabla \boldsymbol{u})^{T}\right) / 2$, and $\ell_{s}$ is the Smagorinsky length scale, modified by Mason \& Thomson's wall damping [17].

\section{Discretization}

Both the forward simulations and the backward simulations are performed in SP-Wind, an in-house research code that was developed in a series of earlier studies on large-eddy simulation and wind-farm simulations, and flow optimization (cf., e.g., [11], [13]-[15]). SP-Wind uses a pseudo-spectral discretization in the horizontal directions. The nonlinear convective terms and the SGS stress are dealiased using the $3 / 2$ rule [22]. Message Passing Interface (MPI) is used to run the simulations in parallel mode, and the FFTW library is employed for Fourier transforms [23]. In the vertical direction, a fourth-order energy-conservative finitedifference discretization is used [24], while time-integration is performed using a classical four-stage fourth-order RungeKutta scheme. A fixed time step corresponding to a CourantFriedrichs-Lewy (CFL) number of approximately 0.4 is used. The time filter (4) is discretized using an implicit Euler method. Finally, for the backward equation, the forward velocity field is required (cf. 11). To this end, the forward field is saved to disk at every time step in the preceding forward simulation around which the adjoint sensitivity is formulated.

An overview of the computational domain is schematically shown in figure 1. Inflow boundary conditions are used for the plane $\Gamma_{1}^{-}$, periodic boundary conditions are used for $\Gamma_{2}^{+}$and $\Gamma_{2}^{-}$, and a symmetry condition is used on $\Gamma_{3}^{+}$. Finallly, a classical high-Reynolds-number wall-stress boundary condition is used on $\Gamma_{3}^{-}$[25], [26] in the forward simulations. For the backward simulations, a related adjoint wall-stress model is used (cf. Ref. [11] for details).

SP-Wind uses a pseudo-spectral discretization in the horizontal directions. Therefore, the inflow boundary condition cannot be straightforwardly implemented as a Dirichlet condition. Instead a fringe-region technique [27] is used that smoothly forces the outflow region in the fringe region towards a desired inlet profile. To this end, we add a fringeforcing term $\lambda(x)\left(\widetilde{\boldsymbol{u}}_{\mathrm{in}}-\widetilde{\boldsymbol{u}}\right)$ to the momentum equation (2), where $\widetilde{\boldsymbol{u}}_{\text {in }}$ is the desired inlet velocity (cf. below), and

$$
\lambda(x)=\lambda_{m}\left[S\left(\frac{x-x_{s}}{d_{s}}\right)-S\left(\frac{x-x_{e}}{d_{e}}+1\right)\right],
$$

where

$$
S(x)= \begin{cases}0 & x \leq 0, \\ {\left[1+\exp \left(\frac{1}{x-1}+\frac{1}{x}\right)\right]^{-1}} & 0<x<1, \\ 1 & x \geq 1,\end{cases}
$$

$x_{s}$ and $x_{e}$ are start and end of the fringe region and $d_{s}$ and $d_{e}$ control the widths of the increasing and decreasing regions in the fringe function $\lambda(x)$.

The turbulent inflow field $\widetilde{\boldsymbol{u}}_{\text {in }}(\boldsymbol{x}, t)$ is generated using a concurrent precursor method [28] in which a separate simulation is concurrently run to the main simulation. This second simulation comprises a classical fully developed boundary-layer simulation (without wind farm) that can be straightforwardly run on periodic domains. The method is visualized in Figure 2, showing velocity snapshots from the precursor boundary layer simulation and the main domain wind-farm simulation, including the coupling from precursor simulation to main-domain fringe region.

Finally, also the adjoint equations require an fringe region approach, since the adjoint boundary condition at $\Gamma_{1}^{+}$corresponds to $\boldsymbol{\xi}=0$. The corresponding fringe-forcing function follows naturally from the forward fringe forcing, leading to $-\lambda(x) \boldsymbol{\xi}$ that is added to (11). 


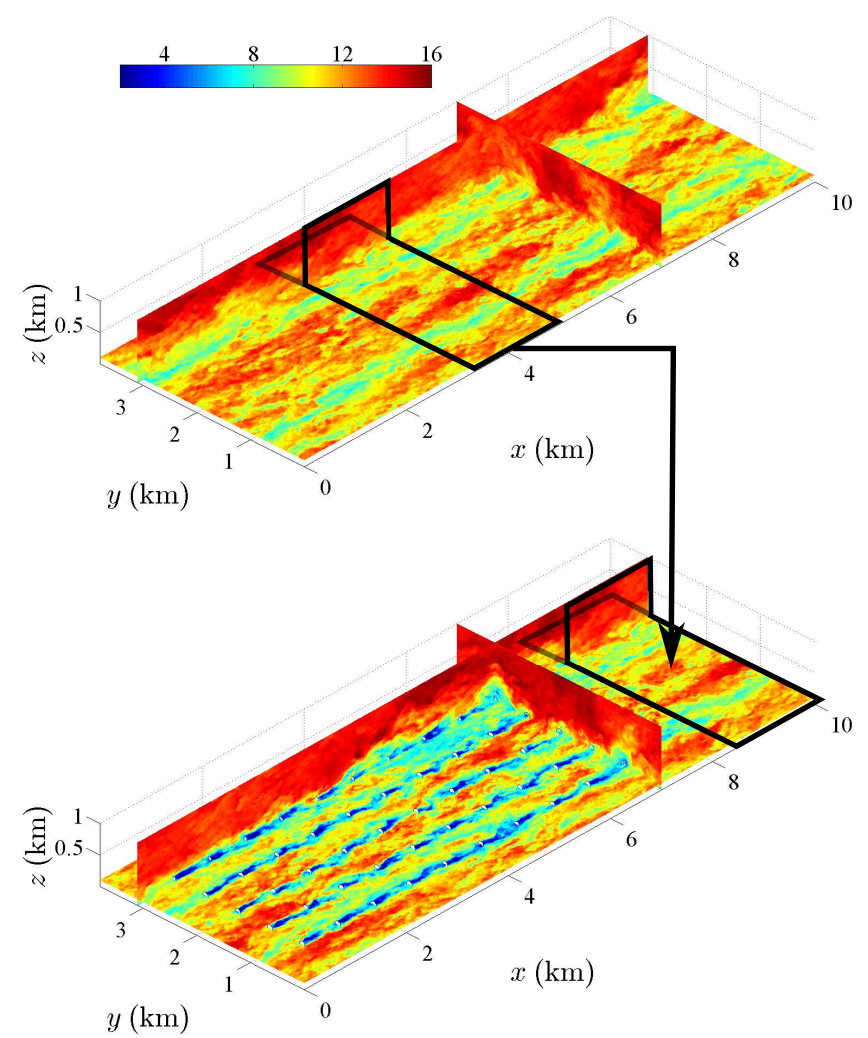

Fig. 2: Snapshots representing instantaneous streamwise velocity fields from the precursor boundary layer simulation (top) and from the finite farm simulation (bottom). The horizontal planes in the figures are taken at the hub height.

TABLE I: Summary of the simulation set-up

\begin{tabular}{ll}
\hline Domain size & $L_{x} \times L_{y} \times H=10 \times 3.8 \times 1 \mathrm{~km}^{3}$ \\
Driving pressure gradient & $f_{\infty}=4 \times 10^{-4} \mathrm{~m} / \mathrm{s}^{2}$ \\
Turbine dimensions & $D=0.1 H=100 \mathrm{~m}, \quad z_{h}=0.1 H$ \\
Turbine arrangement & $10 \times 5$ \\
Turbine spacing & $S_{\mathrm{x}}=7 D$, and $S_{\mathrm{y}}=6 D$ \\
Surface roughness & $z_{0}=10^{-4} H=0.1 \mathrm{~m}$ \\
Grid size & $N_{x} \times N_{y} \times N_{z}=384 \times 256 \times 200$ \\
Time step & $0.6 \mathrm{~s}$ \\
\hline
\end{tabular}

\section{CASE SET-UP}

In the current study, we consider a domain size that corresponds to $L_{x} \times L_{y} \times H=10 \times 3.8 \times 1 \mathrm{~km}^{3}$. The computational grid $N_{x} \times N_{y} \times N_{z}=384 \times 256 \times 200$, using $576 \times 384 \times 200$ when applying the $3 / 2$-dealiasing rule. The fringe region accounts for $15 \%$ of the streamwise length and is located at the downstream end of the domain, starting from $x=8.5 \mathrm{~km}$. Fifty turbines with diameter $D=100 \mathrm{~m}$ are arranged with streamwise spacing $S_{\mathrm{x}}=7 D$, and spanwise spacing $S_{\mathrm{y}}=6 D$, leading to a matrix of 10 by 5 turbines. In the spanwise direction, the spacing between the most left and most right turbine column to the respective left and right boundaries corresponds to $7 D$. This results in a blockage of the wind-farm frontal area to the total frontal area of the domain of around $9 \%$. The inflow velocity is generated in a separate precursor simulation of the boundary layer, which has a domain size and grid resolution identical to those of the actual wind-farm simulation (cf. Figure 2 and discussion above). It is driven by a constant pressure gradient and has periodic boundary conditions in the horizontal directions. Set-up parameters are summarized in Table I.

For the optimal control, we take a time horizon $T=240 \mathrm{~s}$. This roughly corresponds to the time for the flow to pass 4 turbine rows, similar to the value used in Ref. [11]. The optimization algorithm is started with $C_{T, i}^{\prime}(t)=2.0$ (i.e., $\varphi^{(0)}=2.0$ ) for all the turbines in the farm. This corresponds to the optimal operating condition of a lone-standing turbine following the Betz theory. To limit the computational cost, the optimization is not formally converged, but terminated after four conjugate gradient iterations. One conjugate gradient iteration requires roughly 8 standard LES simulations (for the line search), and one adjoint LES for the determination of the gradient, leading to a total of 36 simulations per optimal control window. As soon as the optimization over the first time horizon $[0, T]$ is completed, the optimal controls are used to advance the flow. In standard receding-horizon optimal control, this is done for one time step, after which a new optimization problem is defined for the next time horizon $[\Delta t, T+\Delta t]$. In view of the excessive computational resources required for the optimization, we instead advance the solution for $T_{A}=T / 2$. Subsequently, a new optimization is performed over $\left[T_{A}, T+T_{A}\right]$. We repeat this for 17 optimal control windows, leading to a total control time of $17 T_{A}=2040 \mathrm{~s}$, and a total number of 612 simulations.

Finally, similar to Goit \& Meyers [11], we impose box constraints on the controls, i.e. $0 \leq C_{T, i}^{\prime}(t) \leq 4$. These constraints are trivially applied in the conjugate gradient algorithm, and not explicitly added in the description above. The lower constraint prevents that the turbine operates as a fan. The upper boundary is imposed to avoid $C_{T}^{\prime} \rightarrow \infty$, which is not very practicable from a turbine-construction point of view. However, we do not a priori want to limit $C_{T}^{\prime}$ to the Betz limit $\left(C_{T}^{\prime}=2\right)$. Therefore, we select an adhoc limit of $C_{T}^{\prime}=4$, which, e.g., corresponds to a wind turbine that is constructed with double blade-chord lengths compared to Betz-optimal blade design (cf. Ref [11] for further discussion).

\section{RESULTS}

Goit \& Meyers [11] showed for a fully developed windfarm boundary layer (the so-called 'infinite' wind-farm case) that energy extraction can be increased by up to $16 \%$ by dynamic induction control. The main mechanism was related to improved wake recovery by increasing wake mixing. This was realized by optimal thrust coefficients that were slightly anti-correlated in time with the incoming velocity, thus increasing turbulence levels in the wake. Here, we are studying a similar case in which entrance effects play an important role by changing the boundary conditions, using a precursor simulation in combination with a fringe-region approach. Apart from that, simulation parameters are the same (same number of turbines, arrangement pattern, surface 


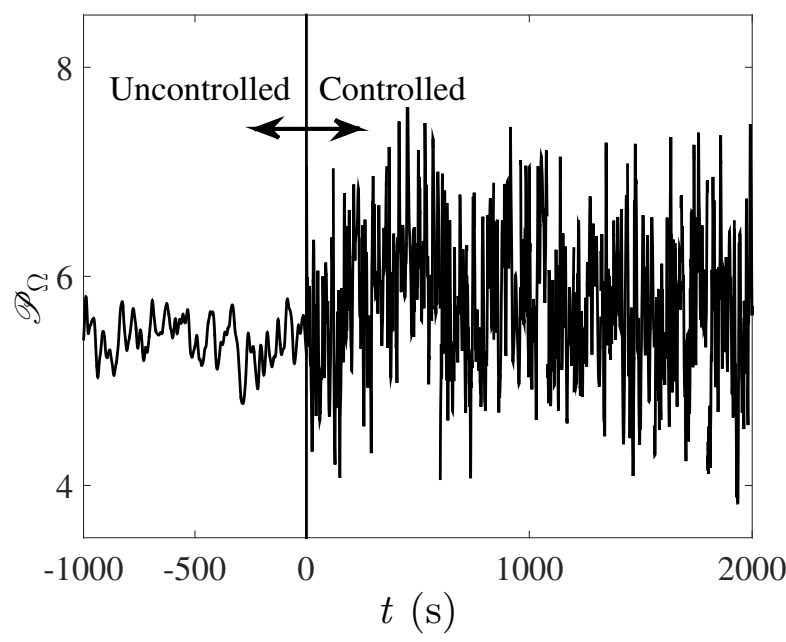

Fig. 3: Time evolution of total farm power output.

roughness, etc.). A typical wind-farm simulation output is appreciated in Figure 2.

In Figure 3, the instantaneous power output of the wind farm is displayed before and after the optimal control is switched on. It is observed that also for this case, windfarm power extraction increases. We observe a gain of $7 \%$ when averaging over the 2000 seconds of optimal control, compared against the greedy case $\left(C_{T}^{\prime}=2\right)$ run over the same time interval. This value is considerably lower than for the infinite case, but still quite significant.

The power extraction over the different turbine rows is further shown in Figure 4. In this figure, it is observed that the average power extraction of the first row decreases, but this is compensated for by the increase in power extraction in the later turbine rows. This is in sharp contrast to static set-point optimization, for which downrating of the first row does not work [29]. In the current study, we further confirmed this by also running our LES using static set points obtained by averaging the dynamic $C_{T}^{\prime}$ values for the different turbine rows. We found that removing the dynamics in the signal, leads to power output that is worse than the uncontrolled case (results not shown here).

The mechanism that improves power extraction is similar to that observed for the fully developed case, i.e. improved wake recovery. This is illustrated in Figure 5, where the hub-height centerline velocity through the turbines is plotted versus the streamwise distance. It is appreciated that the mean velocity recovers much faster in the controlled case; in particular after the first turbine row, this is very pronounced.

Similar to Goit \& Meyers [11], we analyze the correlation between the control signal $C_{T}^{\prime}$, and the turbine disk velocity. To this end, a Reynolds decomposition of the power output (cf. Eq.(6)) is performed. Thus, the control is decomposed in its time mean and fluctuating part, i.e., $C_{T, i}^{\prime} \equiv \overline{C_{T, i}^{\prime}}+$ $\Delta\left[C_{T, i}^{\prime}\right]$. Similarly, $\widehat{V}_{i}^{2} V_{i} \equiv{\widehat{\widehat{V}_{i}^{2}}}_{i}+\Delta\left[\widehat{V}_{i}^{2} V_{i}\right]$. Hence,

$$
P_{r}=\sum_{i=1}^{N_{r}} \frac{1}{2} \overline{C_{T, i}^{\prime}} \overline{\widehat{V}_{i}^{2} V_{i}} A+\sum_{i=1}^{N_{r}} \frac{1}{2} \Delta\left[C_{T, i}^{\prime}\right] \Delta\left[\widehat{V}_{i}^{2} V_{i}\right] A,
$$

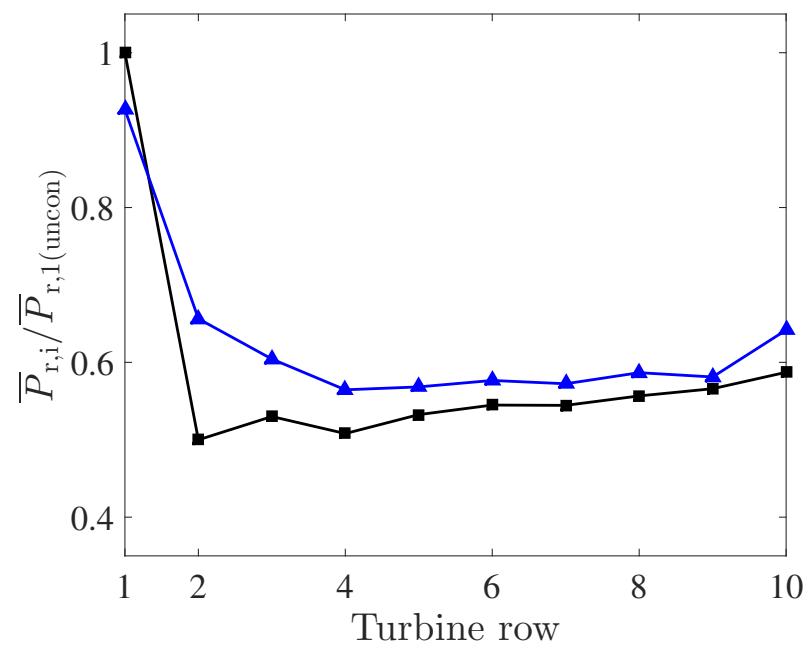

Fig. 4: Comparison of the time averaged power output for the controlled and uncontrolled farm as a function of turbine row. $(\square)$ : power output for the uncontrolled case; $(\boldsymbol{\Delta})$ : power output for optimal control case.

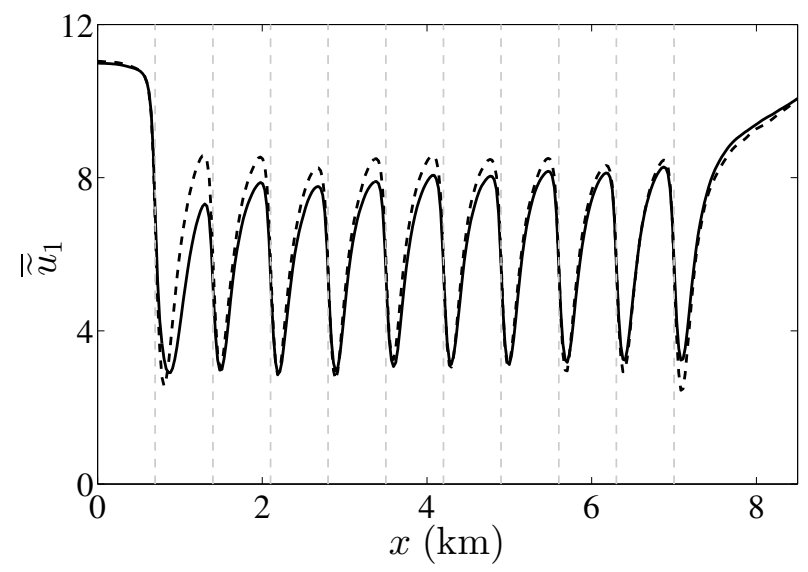

Fig. 5: Time and row averaged profiles of streamwise velocity through the rotor center. ( - , black): uncontrolled case; (---, dashed): optimal control case averaged over the time interval $\left[0,17 T_{A}\right]$. Vertical dashed lines (light grey) represent the location of the turbines.

where $P_{r}$ is the total power output from a turbine row and $N_{r}$ is the number of turbines in the row. It is obvious that the second term on the right-hand side is zero in the uncontrolled case, since $C_{T}^{\prime}$ is constant in that case, so that $\Delta\left[C_{T, i}^{\prime}\right]=0$.

In Figure 6, results are shown for the Reynolds decomposition of the wind-farm power. We find that $C_{T}^{\prime}$ is anticorrelated with the turbine disk velocity. In particular in the first row, this anticorrelation is around $15 \%$. This is much higher than the $6 \%$ found for the infinite farm in Ref. [11]. However, further downstream the amount of anticorrelation drops, and approaches the infinite case. For the last row, the control is not anymore anticorrelated with disk velocity, as increased wake mixing downstream of the last row is not relevant for increased energy extraction. 


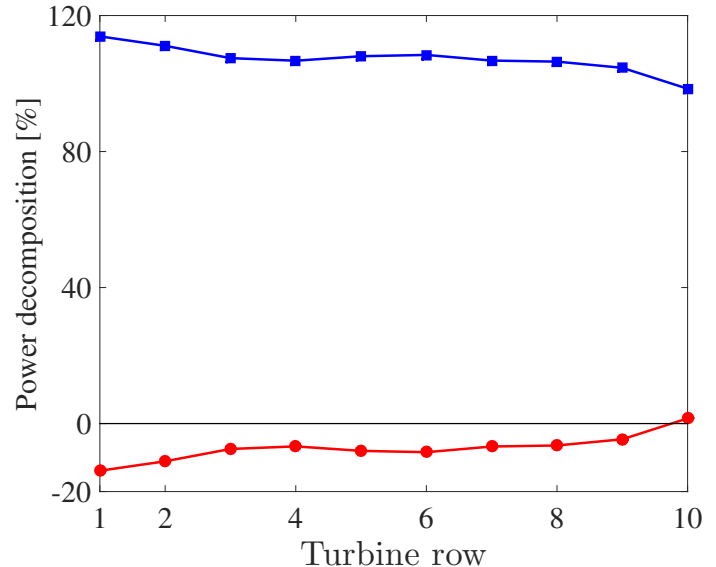

Fig. 6: Reynolds decomposition of power output from different turbine rows. ( $\square$ ): Ratio of the mean component to the total extracted power; $(\bullet)$ : Ratio of the fluctuating component due to $C_{T}^{\prime}$ to the total extracted power.

\section{CONCLUSIONS}

We presented an optimization framework that allows for the optimization of wind-farm control taking into account the full coupling between control actions and the threedimensional turbulent flow. To this end, LES of windfarm boundary layers is combined with the adjoint LES equations into a gradient-based optimization framework. The approach was used to study dynamic induction control of wind turbines in a wind farm in which entrance effects play an important role, with the aim to increase overall energy extraction. Currently, ongoing research is investigating optimal control in different wind-turbine arrangement patterns, optimal control using other wind-turbine models, such as the actuator-line model, and the effect on results of different constraints on the controls. An additional aspect that is of importance for the control to be feasible is the rate of change of the power set points, as high rates of change lead to large additional loading cycles of the turbine. Therefore, in ongoing research we are also looking into the effect of rateof-change limitations on control effectiveness.

\section{REFERENCES}

[1] R. Barthelmie, O. Rathmann, S. T. Frandsen, K. Hansen, E. Politis, J. Prospathopoulos, K. Rados, D. Cabezón, W. Schlez, J. Phillips, A. Neubert, J. G. Schepers, and S. P. van der Pijl, "Modelling and measurements of wakes in large wind farms," J. Physics: Conference Series, vol. 75, p. 012049, 2007.

[2] R. Barthelmie, S. Pryor, S. Frandsen, K. Hansen, J. G. Schepers, K. Rados, W. Schlez, A. Neubert, L. E. Jensen, and S. Neckelmann, "Quantifying the impact of wind turbine wakes on power output at offshore wind farms," J. Atmos. Oceanic Technol., vol. 27, pp. 13021317, 2010.

[3] M. Steinbuch, W. W. de Boer, O. H. Bosgra, S. Peters, and J. Ploeg, "Optimal control of wind power plants," J. Wind Eng Indust. Aerodyn., vol. 27, pp. 237-246, 1988.

[4] K. E. Johnson and N. Thomas, "Wind farm control: addressing the aerodynamic interaction among wind turbines," in American Control Conference, 2009. ACC '09. St. Louis, MO: IEEE, June 2009, pp. 2104-2109.
[5] T. Horvat, V. Spudic, and M. Baotic, "Quasi-stationary optimal control for wind farm with closely spaced turbines," in MIPRO, 2012 Proceedings of the 35th International Convention, 2012, pp. 829-834.

[6] M. Soleimanzadeh, R. Wisniewski, and S. Kanev, "An optimization framework for load and power distribution in wind farms," $J$. Wind Eng. Ind. Aerodyn., vol. 107-108, pp. 256-262, 2012.

[7] T. Knudsen, T. Bak, and M. Svenstrup, "Survey of wind farm controlpower and fatigue optimization," Wind Energy, 2014.

[8] J. Annoni, P. Gebraad, A. Scholbrock, P. Fleming, and J. van Wingerden, "Analysis of axial-induction-based wind plant control using an engineering and a high-order wind plant model," Wind Energy, p. In Press, 2015.

[9] M. Soleimanzadeh, R. Wisniewski, and A. Brand, "State-space representation of the wind flow model in wind farms," Wind Energy, vol. 17, no. 4, pp. 627-639, 2014.

[10] M. Soleimanzadeh, R. Wisniewski, and K. Johnson, "A distributed optimization framework for wind farms," Journal of Wind Engineering and Industrial Aerodynamics, vol. 123, Part A, pp. 88 - 98, 2013.

[11] J. P. Goit and J. Meyers, "Optimal control of energy extraction in wind-farm boundary layers," Journal of Fluid Mechanics, vol. 768, pp. 5-50, 2015.

[12] M. Calaf, C. Meneveau, and J. Meyers, "Large eddy simulation study of fully developed wind-turbine array boundary layers," Phys. Fluids, vol. 22, p. Art no 015110, 2010.

[13] J. Meyers and P. Sagaut, "Evaluation of Smagorinsky variants in largeeddy simulations of wall-resolved plane channel flows," Physics of Fluids, vol. 19, no. 095105, 2007.

[14] S. Delport, M. Baelmans, and J. Meyers, "Constrained optimization of turbulent mixing-layer evolution," J. Turbul., vol. 10, no. 18, pp. $1-26,2009$.

[15] J. Meyers and C. Meneveau, "Large eddy simulations of large wind-turbine arrays in the atmospheric boundary layer," 48th AIAA Aerospace Sciences Meeting Including the New Horizons Forum and Aerospace Exposition, Orlando, Florida, vol. AIAA 2010-827, pp. 1$10,2010$.

[16] J. Smagorinsky, "General circulation experiments with the primitive equations: I. The basic experiment," Monthly Weather Review, vol. 91, no. 3, pp. 99-165, 1963.

[17] P. J. Mason and T. J. Thomson, "Stochastic backscatter in large-eddy simulations of boundary layers," Journal of Fluid Mechanics, vol. 242, pp. 51-78, 1992.

[18] J. Meyers and C. Meneveau, "Flow visualization using momentum and energy transport tubes and applications to turbulent flow in wind farms," Journal of Fluid Mechanics, vol. 715, pp. 335-358, 2013.

[19] W. H. Press, S. A. Teukolsky, W. T. Vetterling, and B. P. Flannery, Numerical Recipes in FORTRAN77: The art of scientific computing. Cambridge University Press, 2nd edition, 1996.

[20] D. G. Luenberger, Linear and Nonlinear Programming, 2nd ed. Kluwer Academic Publishers, 2005.

[21] J. Nocedal and S. J. Wright, Numerical Optimization, 2nd ed. Springer, 2006.

[22] C. Canuto, M. Y. Hussaini, A. Quarteroni, and T. A. Zang, Spectral Methods in Fluid Dynamics. Springer, 1988.

[23] M. Frigo and S. G. Johnson, "The design and implementation of FFTW3," Proceedings of the IEEE, vol. 93, no. 2, pp. 216-231, 2005, special issue on "Program Generation, Optimization, and Platform Adaptation".

[24] R. W. C. P. Verstappen and A. E. P. Veldman, "Symmetry-preserving discretization of turbulent flow," Journal of Computational Physics, vol. 187 , pp. 343-368, 2003.

[25] C.-H. Moeng, "A large-eddy simulation model for the study of planetary boundary-layer turbulence," J. Atmos. Sci., vol. 41, pp. $2052-$ 2062, 1984.

[26] E. Bou-Zeid, C. Meneveau, and M. B. Parlange, "A scale-dependent Lagrangian dynamic model for large eddy simulation of complex turbulent flows," Physics of Fluids, vol. 17, no. 025105, 2005.

[27] P. R. Spalart, "Direct numerical study of leading edge contamination," in Fluid Dynamics of Three-Dimensional Turbulent Shear Flows and Transition, ser. AGARD Conference Proceedings, vol. 438, 1988, pp. 5.1-5.13.

[28] R. J. Stevens, J. Graham, and C. Meneveau, "A concurrent precursor inflow method for large eddy simulations and applications to finite length wind farms," Renewable Energy, vol. 68, pp. 46-50, 2014.

[29] P. M. O. Gebraad, "Data-driven wind plant control," Ph.D. dissertation, Delft University of Technology, 2014. 